\title{
ATENÇÃO AO NEONATO NA ESTRATÉGIA SAÚdE DA FAMÍLIA: AVANÇOS E DESAFIOS PARA A ATENÇÃO INTEGRAL*
}

\author{
Maria Aparecida Munhoz Gaiva ${ }^{1}$, Naudia da Silva Dias ${ }^{2}$,Valéria de Carvalho Araújo Siqueira ${ }^{3}$
}

\begin{abstract}
RESUMO: Estudo descritivo-exploratório de abordagem qualitativa analisou avanços e desafios na atenção ao neonato com a implantação de unidade de saúde da família em Cuiabá - Mato Grosso. Os dados foram coletados por meio de entrevista e observação participante e submetidos à análise de conteúdo temática. Os sujeitos foram um médico, um enfermeiro, dois técnicos em enfermagem, quatro agentes comunitários, e seis mães de neonatos em acompanhamento no momento da coleta de dados. Dentre os avanços promovidos pela Estratégia Saúde da Família está a melhoria do acesso e do vínculo entre usuários e profissionais e da resolutividade da atenção. A efetivação do trabalho em equipe e a participação da família na assistência são desafios a serem superados pela equipe para prestar atenção integral ao neonato. Conclui-se, na perspectiva da integralidade, que apesar dos avanços, faz-se necessária maior valorização e incentivo à participação da família no processo de cuidar.

PALAVRAS-CHAVE: Atenção básica; Programa saúde da família; Assistência integral à saúde; Recém-nascido.

\section{ATTENTION TO THE NEWBORN IN THE FAMILY HEALTH STRATEGY: ADVANCES AND CHALLENGES FOR INTEGRAL CARE}

\begin{abstract}
This descriptive-exploratory study with a qualitative approach analyzed advances and challenges in care for the newborn resulting from the implantation of a family health center in Cuiabá in Mato Grosso. The data was collected through interviews and participant observation and was submitted to thematic content analysis. The subjects were a doctor, a nurse, two nurse technicians, four community health workers, and six mothers of newborns being monitored at the time of the data collection. Among the advances promoted by the Family Health Strategy is the improvement of access and of the link between service users and health professionals and of the solution of the care. The establishment of the team work and the participation of the family in the care are challenges to be overcome by the team in providing integral care to the newborn. It is concluded that from the perspective of integrality, in spite of the advances, greater valuing and incentivizing of the participation of the family in the care process is needed.

KEYWORDS: Primary care; Family health program; Integral health care; Newborn.

\section{ATENCIÓN AL NEONATO EN LA ESTRATEGIA SALUD DE LA FAMILIA: AVANCES Y DESAFÍOS PARA LA ATENCIÓN INTEGRAL}

RESUMEN: Estudio descriptivo-exploratorio de abordaje cualitativo que há analizado avances y desafíos en la atención al neonato con la implantación de unidad de Salud de la Familia en Cuiabá Mato Grosso. Los datos fueron obtenidos por medio de entrevista y observación participante y sometidos al análisis de contenido temático. Los sujetos fueron un médico, un enfermero, dos técnicos en enfermería, cuatro agentes comunitarios, y seis madres de neonatos en acompañamiento en el momento de recoger los datos. Entre los avances promovidos por la estrategia Salud de la Familia, hay la mejoría del acceso y del vínculo entre usuarios y profesionais y de la resolutividad de la atención. La efectivación del trabajo en equipo y la participación de la familia en la asistencia son desafíos a superar por el equipo para prestar atención integral al neonato. Se concluiye que, enla perspectiva de la integralidad, a pesar de los avances, es necesaria mayor valoración y incentivo a la participación de la familia en el proceso de cuidar.

PALABRAS CLAVE: Atención básica; Programa salud de la familia; Asistencia integral a la salud; Recién nacido.

\footnotetext{
* Artigo extraído da dissertação "Assistência ao neonato em uma unidade do Programa Saúde da Família do município de Cuiabá-MT" apresentada à Universidade Federal de Mato Grosso em 2010.

${ }^{1}$ Enfermeira. Doutora em Enfermagem. Professora do Programa de Pós-Graduação da Faculdade de Enfermagem da Universidade Federal de Mato Grosso - UFMT. Pesquisadora do CNPq.

${ }^{2}$ Enfermeira. Mestre em Enfermagem. Professora do Curso de Graduação em Enfermagem da Universidade do Estado do Mato Grosso. ${ }^{3}$ Enfermeira. Mestre em Enfermagem. Professora do Curso de Graduação em Enfermagem do Centro Universitário de Várzea Grande-MT.
}

Autor correspondente:

Maria Aparecida Munhoz Gaiva

Universidade Federal de Mato Grosso

Rua General Valle, 431 - 78010-000 - Cuiabá-MT-Brasil

Email: mamgaiva@yahoo.com.br
Recebido: $15 / 04 / 2012$

Aprovado: 28/11/2012

Cogitare Enferm. 2012 Out/Dez; 17(4):730-7 


\section{INTRODUÇÃO}

Nos primeiros anos de vida da criança as mudanças decorrentes do desenvolvimento humano são mais intensas e visíveis. Por se tratar de um período tão dinâmico da vida, a atenção à saúde da criança tem representado um campo prioritário dentro dos cuidados à saúde das populações. Neste contexto, no Brasil, ao longo das últimas décadas, as ações voltadas para a atenção à criança no primeiro ano de vida estiveram no escopo das políticas públicas de saúde ${ }^{(1)}$.

Na década de 1970, houve a implantação do Programa de Saúde Materno-Infantil (PSMI), cujas ações estavam, basicamente, voltadas para o acompanhamento do pré-natal, controle dos partos domiciliares e do puerpério, principalmente em relação à qualidade da assistência pré-natal; poucas eram as ações voltadas à criança ${ }^{(2)}$. Posteriormente, em 1984, surgiu o Programa de Atenção Integral à Saúde da Criança (PAISC) que objetivou o controle das principais morbidades na infância e a redução da mortalidade infantil ${ }^{(2)}$.

Em 2004 o Ministério da Saúde lançou a Agenda de Compromissos para a Saúde Integral da Criança e Redução da Mortalidade Infantil, que ressalta a organização da assistência a essa população. Este documento destaca, entre as linhas de cuidado, a atenção integral à saúde da criança, a redução da mortalidade infantil, as ações de saúde da mulher com atenção humanizada e qualificada à gestante e ao recém-nascido, e o incentivo ao aleitamento materno desde a gestação até o puerpério ${ }^{(3)}$.

Assim, a assistência à saúde da criança tem se transformado em decorrência de mudanças nos perfis epidemiológico e demográfico da população infantil. Além disso, os avanços científicos e a incorporação de tecnologias, a participação das mães e, muitas vezes, dos pais na assistência, o trabalho multiprofissional e a preocupação com a qualidade de vida têm contribuído, significativamente, para mudança do olhar sobre a saúde da criança. Considera-se a criança como um todo e em suas múltiplas relações, a família é valorizada como também o contexto em que a criança vive. Destacam-se princípios como o acesso aos serviços de saúde e a integralidade do cuidado nos diversos níveis de atenção ${ }^{(4)}$.

A atenção integral foi definida na Constituição Federal de 1988 como uma das diretrizes do Sistema Único de Saúde (SUS). Consiste em priorizar as ações preventivas, sem desconsiderar as curativas, abrangendo o individual e o coletivo, no intuito de melhorar a qualidade de vida da população ${ }^{(5)}$.
Com a criação, na década de 1990, da Estratégia Saúde da Família (ESF), ampliaram-se as ações voltadas para a atenção à criança no âmbito da atenção básica. A assistência à saúde da criança/neonato, na atual organização da atenção básica, está sustentada nos princípios do SUS e da ESF e toma como direcionalidade as proposições da Agenda de Compromisso para a Saúde Integral da Criança e Redução da Mortalidade Infantil. Neste sentido, são enfocados os seguintes eixos norteadores: acesso universal, ações intersetoriais, acolhimento, assistência integral e resolutiva, equidade, trabalho em equipe, ações de promoção de saúde e participação da famíliaa ${ }^{(3)}$.

A assistência à criança na ESF tem por objetivos contemplar a promoção à saúde, a prevenção de agravos e a vigilância em saúde, por meio da educação em saúde às crianças, aos pais e seus familiares. E também, por meio de orientações antecipatórias aos riscos de agravos em saúde, oferecendo medidas preventivas mais eficazes, dando prioridade às medidas de promoção à saúde, ao invés de tratamento das doenças ${ }^{(6)}$.

$\mathrm{Na}$ ESF, em nossa realidade, o que vem sendo praticado pelos serviços na atenção ao neonato são ações clínicas individuais, como a consulta médica e de enfermagem; as visitas domiciliares à família e à criança, merecendo destaque o recém-nascido na primeira semana de vida. Ainda, imunização, acompanhamento do crescimento e desenvolvimento por meio das consultas e do controle do peso e atividades educativas, dentre outras $^{(7)}$; ações essas que contribuem para melhorar os índices de morbimortalidade e qualidade de vida.

Considerando a assistência ao neonato na atenção básica e a necessidade de cuidado orientado pela integralidade, temos o pressuposto que, apesar de as proposições atuais da ESF orientar que a organização do trabalho assistencial deve estar centrada no atendimento às necessidades do sujeito no contexto da família, a assistência prestada ao neonato nas Unidades de Saúde da Família (USF), em nossa realidade, ainda é focada no atendimento aos aspectos clínicos e nas queixas maternas. A ênfase da atenção é o crescimento e desenvolvimento da criança e a família não é objeto de atenção dos profissionais e não tem suas necessidades consideradas no processo assistencial.

Ponderando que a criança, no primeiro mês de vida, está mais vulnerável a determinados agravos de saúde, e que seu acompanhamento é de suma importância na vida do bebê e da mãe, e que as unidades básicas constituem porta de entrada para o acesso aos serviços de saúde, este estudo objetivou analisar os avanços e desafios na atenção ao neonato com a implantação de uma USF no município de Cuiabá - Mato Grosso. 


\section{MÉTODO}

O presente estudo, do tipo descritivo-exploratório, foi desenvolvido com base na abordagem qualitativa de pesquisa, tendo como cenário uma USF, no município de Cuiabá - Mato Grosso. Os dados apresentados neste artigo são parte de uma pesquisa cujo objeto foi a organização da atenção à saúde do neonato ${ }^{(8)}$.

A escolha da unidade para a realização da pesquisa foi baseada nos seguintes critérios: equipe de saúde com atuação há pelo menos 12 meses na mesma unidade; cobertura pré-natal acima de $95 \%$ e com pelo menos 10 neonatos inscritos e em acompanhamento na unidade no momento da coleta de dados.

A coleta dos dados foi realizada, em 2008, por meio de entrevista com 8 trabalhadores, sendo um médico, um enfermeiro, dois técnicos em enfermagem e quatro agentes comunitários (ACS). As entrevistas foram direcionadas a partir da seguinte questão norteadora: O que mudou na assistência ao neonato com a implantação da unidade de saúde da família? Foram também entrevistas seis mães de neonatos em acompanhamento na unidade. Foi utilizado como requisito para encerramento da coleta de dados o critério de saturação, que prevê o término da coleta quando as informações começam a se tornar repetitivas ${ }^{(9)}$.

Utilizamos também, como estratégia de coleta de dados, a técnica da observação participante, com o intuito de captar as diferentes ações/atividades que os profissionais desenvolviam na atenção ao neonato. Como forma de registro das observações foi utilizado o diário de campo. Nele foram documentadas descrições de interações, diálogos e reflexões suscitadas no processo da pesquisa que permitiram conhecer a organização da assistência ao neonato no contexto da unidade.

Para a análise dos dados coletados foram adotadas as diretrizes do método qualitativo: ordenação, classificação dos dados, síntese e análise final ${ }^{(9)}$. Emergiram dos dados as seguintes categorias temáticas: Mudanças na assistência ao neonato com a implantação da USF e Desafios para prestar assistência integral ao neonato e família.

O projeto de pesquisa foi submetido ao Comitê de Ética em Pesquisa do Hospital Universitário Júlio Müller e aprovado pelo Parecer n. 340/CEP-HUJM/2007.

\section{RESULTADOS}

\section{Mudanças na assistência ao neonato com a implan- tação da USF}

Em relação aos avanços, profissionais e mães elencaram vários aspectos, dentre eles, a melhoria do acesso à atenção à saúde, já que não existia um serviço de saúde no bairro, como no depoimento a seguir:

Quando não tinha [ESF] nós tínhamos que sair daqui para ir para uma policlínica, e agora ficou mais fácil, você vem aqui! Você já vem consultar, já pega o encaminhamento, prá depois ir fazer! Tem também a consulta de rotina, faz o crescimento e desenvolvimento da criança, tem a vacinação, a consulta, o dia a dia da criança. Eu achava que um dia ia ter esse tipo de atendimento e hoje, graças a Deus, tem para não ter que estar indo prá longe com o bebê. (Mãe 1)

A implantação de uma USF na comunidade possibilitou maior vínculo dos usuários com os profissionais, ou vice-versa, como evidenciado pelo trabalhador:

[...] um vínculo de maior proximidade, né? Você conhece melhor a sua clientela e a clientela também te conhece, tanto que a maior parte de nossos clientes a gente conhece pelo nome. Eles também sabem o nosso nome! (ACS 1)

A proximidade dos profissionais de saúde com a população faz com que haja maior conhecimento das reais necessidades dos usuários, levando-os a participar de forma ativa na busca de soluções para os problemas que podem estar afetando a sua saúde, como a seguir:

Na ESF a gente trabalha muito lá dentro da casa da familia, vocêfica sabendo de tudo [...] passando orientações de vida. É um programa diferenciado, porque a gente tem acesso livre na casa dos pacientes, a gente já vira até comadres! Porque a gente acaba sabendo da vida dele! (Técnica em enfermagem 1)

Em relação à assistência ao neonato na unidade, existe um dia específico para atendimento, o que facilitou para as mães, que já não precisam acordar de madrugada, para conseguir consulta para o filho. Além do mais, caso a criança necessite de atendimento fora do dia da consulta ela será assistida sem agendamento prévio de acordo com o depoimento a seguir: 
O acompanhamento da criança é mais próximo, porque a criança, ela tem prioridade em nossa unidade! A mãe, qualquer coisa que o bebê tem, ela pode vir, pode estar conversando, não somente com a recepcionista, mas comigo, ou com o enfermeiro ou com o médico [...]. Aqui tem facilidades, porque elas têm livre acesso, elas vêm ao encontro de suas necessidades e o acompanhamento dos bebês é agendado. (Técnica de enfermagem 2)

Para as mães, a USF além de melhorar o acesso ao atendimento de saúde, tornou-o mais resolutivo, de acordo com a seguinte fala:

Tem mais atenção pro bebê! Aqui ficou mais perto! Tem mais atenção! A criança fica doente, logo já traz e já é resolvido. Tem um dia só pra elas, para as crianças, só pediátrico, foi bom assim. [...] ai a gente pode estar retornando no dia marcado no cartão, isso também mudou bastante e ficou bom. (Mãe 5)

Ao indagarmos sobre o que mudou na atenção ao neonato/bebê com a criação da USF, identificamos que, para os profissionais, a mudança se pauta no acompanhamento do crescimento e desenvolvimento, vacinação e orientações para o aleitamento materno:

Esse trabalho de $C D$ [crescimento e desenvolvimento]! De acompanhamento da criança, quando não tinha o $P S F$, não tinha esse acompanhamento de $C D$ [...]. E com a abertura da ESF esse trabalho foi ampliado e deu oportunidade para todos, todos podem vir, principalmente com a nossa orientação de cada mês [...]. (Médico)

As crianças são acompanhadas em seu CD [crescimento e desenvolvimento], temos um cronograma de mês a mês. Trabalhamos na perspectiva que a mãe não traga a criança na unidade somente quando ela está doente, mas que ela venha à unidade por causa do compromisso vacinal, do desenvolvimento, da higiene e da alimentação [...]. (Enfermeiro)

A visita domiciliar é vista pelos participantes como um instrumento para a prestação de assistência à saúde da família, especialmente para o acompanhamento dos neonatos:

Além de todo acompanhamento mensal da criança, a gente pede para o ACS passar toda semana na casa para ver se a criança está bem, mamando no peito, se a mãe tá dando mamadeira, chazinho [...]. (Enfermeiro)
Em decorrência da presença mais constante dos ACS, especialmente nas visitas domiciliares, a relação destes com a família é mais próxima e a mãe considera um aspecto positivo a relação estabelecida, como refletem os depoimentos:

A agente vem procurar porque a gente não foi no posto [...] eu gosto disso, isso é positivo para mim! É bom que eles [ACS] estão acompanhando a gente. (Mãe 5)

Os agentes vêm dar informação prá gente, tudo o que se pergunta do bebê eles já sabem, tudo! (Mãe 1)

\section{Desafios para prestar assistência integral ao neonato e família}

Apesar de a família ser o objeto de atenção da ESF, os profissionais reconhecem que esta ainda é pouco valorizada pela equipe e que há necessidade de incentivo à sua participação no processo assistencial na unidade:

A gente vê mais a mãe participando do acompanhamento da criança, poucos são os pais que acompanham. Talvez a gente tenha que fazer um trabalho de incentivo, ir às residencias e levantar a necessidade de envolvimento da família, que não é só uma responsabilidade da mãe, precisa da cumplicidade do pai, do envolvimento dos avós e daqueles que cuidam da criança. (Enfermeiro)

A participação da familia na atenção à criança é importantissima! A gente até pede para vir pai, mãe, avó, todo mundo na consulta. (Médico)

A despeito de a ESF propor a viabilização do trabalho de forma interdisciplinar e em equipe, integrando áreas técnicas e profissionais de diferentes formações, detectamos através das falas de alguns profissionais que a efetivação do trabalho em equipe é um dos desafios a ser superado para que seja possível prestar assistência integral ao neonato:

A gente vai ter que tentar trabalhar mais em equipe, e a iniciativa não é só do enfermeiro, tem que ser também de vontade médica, da equipe, dos técnicos em enfermagem, que também precisam ter uma clareza maior da visão do trabalho, um esclarecimento melhor, o porquê da prioridade de trabalhar melhor com o recém-nascido! (Enfermeiro) 
Se o trabalho fosse mais extenso, se a gente começasse a trabalhar em grupo, em equipe mesmo, a gente faria muito mais. O que falta é um incentivar o outro! (Técnico em enfermagem 2)

Os discursos dos profissionais, exemplificado abaixo, evidenciam a necessidade de construção de um projeto assistencial que seja compartilhado por todos, com articulação entre ações e interação entre os profissionais da equipe. Outro ponto destacado como desafio pelos entrevistados foi a mudança nas relações estabelecidas entre os profissionais e a comunidade:

Porque ESF não é só médico, não é só o enfermeiro, é uma equipe! Todos precisam estar juntos em prol de uma mesma causa. (Médico)

[...] eu acho que para a comunidade seria melhor se ela pudesse ter a mesma intimidade que tem conosco, com toda a equipe [...] é a forma com que eles olham a equipe. Eles acham que a equipe, que o enfermeiro é mais do que eles às vezes [...] a estima deles é baixa! Eles não se vêm na condição de discutirem com o médico [...] se o médico tivesse mais intimidade com ele [usuário] ele viria aqui com maior facilidade. (ACS 1)

Identificamos a dificuldade de estabelecer vínculo entre profissionais e usuários, com o distanciamento nas relações interpessoais interferindo no processo assistencial. Identifica-se que os usuários não se sentem à vontade, ou seja, em situação de igualdade no relacionamento. Porém, essa reação é atribuída ao comportamento assumido pelos profissionais nas relações com a população, como depoimento de ACS:

Mas, diante do médico, não se sentem bem [os usuários], eles não se sentem igual [...]. Eles fazem a pergunta e a resposta é fria, ai eles não perguntam de uma vez! (ACS 2)

Por outro lado, as mães entrevistadas reconhecem a relação com os profissionais como boas, uma vez que são bem tratadas, ouvidas e orientadas, como observa-se no discurso a seguir:

Eu converso com ele [médico], eu pergunto, falo o que eu tenho que falar, ele explica prá mim e fala o que tenho que fazer, me ouve. Eu ouço ele e ele me ouve. (Mãe 4)

Os discursos demonstram o reconhecimento dos usuários como cidadãos portadores de direitos e corresponsáveis pelos cuidados e qualificação da atenção à saúde:

Que a população se conscientize que o PSF é um direito deles. Que participem mais, que cobrem mais a qualidade do atendimento. Que exijam mais dos profissionais e dos órgãos da saúde. (Enfermeiro)

Deixe eles [usuários] saberem que têm o direito de cobrar da unidade uma assistência de qualidade e que participem mais [...]. (ACS 2)

\section{DISCUSSÃO}

O acesso é um dos princípios fundamentais da atenção básica, por

possibilitar acesso universal e contínuo a serviços de saúde de qualidade e resolutivos, caracterizados como a porta de entrada preferencial do sistema de saúde $[\ldots]^{(10: 11)}$.

O acesso universal deve ser entendido como um direito de toda criança em receber assistência de saúde, e é responsabilidade das unidades acolherem todos os que as procuram, propiciando escuta de suas demandas ou problemas de saúde, e avaliação qualificada de cada situação ${ }^{(3)}$.

$\mathrm{O}$ vínculo com os usuários do serviço de saúde amplia a eficácia das ações e favorece a participação dos mesmos durante a prestação da assistência, possibilitando a constituição de sujeitos autônomos, tanto profissionais quanto pacientes, pois não há construção de vínculo sem que o usuário seja reconhecido na condição de sujeito, que fala, julga e deseja ${ }^{(11)}$. Segundo o Ministério da Saúde, a USF não deve perder de vista o usuário, responsabilizando-se pelas referências necessárias, contatando outros serviços, discutindo os casos e recebendo-os de volta, na contrarreferência, para continuar atuando no nível dos cuidados básicos ${ }^{(12)}$.

As diretrizes políticas para a infância têm considerado o período neonatal como prioritário, propondo ações que enfocam o incentivo ao aleitamento materno, vacinação, acompanhamento de crescimento e desenvolvimento, nutrição e prevenção de acidentes ${ }^{(3,13)}$. Na atenção às crianças, especialmente as menores de um ano, a preservação da saúde está também na dependência de ações e cuidados que antecipem a manifestação de doenças, evidenciando assim, a importância do controle do peso, estatura, vacinas, bem como da qualidade da alimentação ${ }^{(14)}$. 
O acompanhamento do crescimento e desenvolvimento é considerado o eixo norteador da atenção integral à saúde da criança e perpassa todas as linhas de cuidados definidas pela Agenda de Compromissos para a Saúde Integral e Redução da Mortalidade Infantil. Este constitui-se um método simples, de baixo custo e de grande eficácia, em que todas as ações de promoção, proteção e recuperação da saúde da população infantil culminam efetivamente em crescimento e desenvolvimento saudável e redução da mortalidade infantil ${ }^{(3)}$

Em um estudo realizado em unidades de saúde no Estado de Pernambuco, cujo objetivo foi analisar a ação de acompanhamento do crescimento de crianças menores de um ano, observou-se elevado percentual de crianças que não apresentavam o peso marcado no gráfico ou que não tinham a curva de crescimento traçada no cartão. Ainda, o estudo revelou que as ações de acompanhamento do crescimento não são realizadas com todas as crianças na primeira consulta, contrariando as recomendações do Ministério da Saúde ${ }^{(15)}$.

A integralidade da atenção ao neonato não se reduz à garantia do acesso e ao acompanhamento do crescimento e desenvolvimento e situação vacinal. Para a criança receber assistência integral, segundo o Ministério da Saúde, é preciso empregar os princípios norteadores do cuidado. Esses são o planejamento e desenvolvimento de ações intersetoriais; acesso universal; acolhimento; responsabilização pela equipe; não restringir a demanda apresentada; prestar uma assistência resolutiva; ter equidade na atenção; atuação em equipe; desenvolvimento de ações coletivas; participação da família e controle social; avaliação permanente e sistematizada ${ }^{(3)}$.

Neste sentido, a ESF propõe inovações na organização e dinâmica da atenção mediante o uso de tecnologias que permitam a aproximação dos profissionais das pessoas, famílias e comunidades, tendo por objetivo a prestação de assistência segundo as reais necessidades da população. Entre as tecnologias utilizadas no trabalho das equipes de saúde da família, encontra-se a prática sistemática das visitas domiciliares $^{(16)}$. Apesar de ser uma tecnologia de cuidado antiga, a visita domiciliar traz resultados inovadores, uma vez que possibilita conhecer o mundo da família, o local onde ela vive, sua rotina diária, sua cultura, seu modo de vida, seus hábitos e seus cuidados de saúde. E, portanto, deve ser realizada por todos os profissionais da equipe de saúde da família possibilitando assim o cuidado integral ao neonato e à família.
A importância do ACS para a comunidade se pauta na identificação dele com as pessoas de sua área de abrangência, pela capacidade de facilitar o vínculo com os demais membros da equipe e pela possibilidade de traduzir os interesses, necessidades e reivindicações da população sob sua responsabilidade ${ }^{(17)}$. A ESF é percebida pelos entrevistados como um modelo de assistência que propõe mudanças em relação ao processo de trabalho da equipe e à prática assistencial. Identificamos nos discursos que o trabalho em saúde da equipe estudada apresenta fragilidade nas relações. Foi possível também captar, através das falas e da observação em campo, que o trabalho em equipe vem reproduzindo a divisão social do trabalho. Percebemos que alguns membros da equipe se sentem prejudicados nesta relação, porém identificam ser necessária uma reorganização do trabalho, com planejamento do trabalho da equipe, rompendo com as características de um trabalho compartimentalizado, com execução de tarefas prescritas e sem planejamento coletivo das necessidades dos sujeitos envolvidos (profissionais e usuários).

O não reconhecimento da importância do trabalho interdisciplinar por parte de membros da equipe de saúde da família gera tensões na prática cotidiana, dificultando o desempenho da equipe e a operacionalização dos pressupostos da $\mathrm{ESF}^{(18)}$. O trabalho das equipes de saúde da família requer a construção de um projeto assistencial comum com complementaridade dos trabalhos especializados, em que os agentes envolvidos estabeleçam interação entre si e com os usuários ${ }^{(19)}$.

$\mathrm{Na}$ avaliação da qualidade dos cuidados recebidos, os usuários levam em consideração, além do desempenho técnico, atitudes como a compreensão, o acolhimento e a comunicação com os profissionais que os assistem ${ }^{(20)}$. No entanto, os processos de inter-relação entre profissionais e usuários, no contexto da saúde, têm sido pautados, muitas vezes, por concepções que permaneceram sedimentadas na tradição de um profissional portador do saber se relacionando com um usuário alienado deste saber com o intuito de educá-lo para um melhor comportamento em saúde. A ESF se pauta no trabalho em equipe multiprofissional e na participação social, á medida que decorrem e expressam as relações entre a população de referência, o serviço e a equipe, bem como as relações diretas entre trabalhadores e usuários. Nesse sentido, uma boa relação entre equipe de saúde e comunidade proporcionará uma melhoria do acesso e da qualidade da atenção à saúde. A Agenda de Compromissos para a Saúde Integral da Criança e Redução da Mortalidade Infantil define a 
participação social como um dos princípios norteadores do cuidado à saúde da criança. Isso se dá por meio do

incentivo à participação da família em toda a atenção à criança, envolvendo-a com a informação sobre os cuidados e problemas de saúde, bem como nas propostas de abordagem e intervenções necessárias entendidas como direito de cada cidadão e potencial de qualificação e humanização de assistência ${ }^{(3: 15)}$.

Assim, considerando que a família deve ser o objeto de atenção da ESF, esta ainda é pouco valorizada pela equipe, havendo a necessidade de reconstrução do cuidado à saúde do neonato, inserindo a família no processo de cuidar, através de uma relação de diálogo e vínculo, articulação dos saberes técnico-científico e saberes práticos/cultural das famílias, na busca cada vez mais do cuidado integral.

\section{CONSIDERAÇÕES FINAIS}

Conhecer a realidade de assistência ao neonato em uma USF, a partir do olhar dos profissionais e das mães, possibilitando-nos aproximar dessa realidade e destacar os desafios e superações a serem empreendidos pela equipe para a construção de uma atenção integral a essa população. Em síntese, tanto profissionais como as mães ponderaram que a implantação da USF melhorou o acesso e a resolutividade da atenção ao neonato, além de propiciar maior vinculo dos usuários com profissionais e unidade de saúde.

Tomados em conjunto, os resultados deste estudo evidenciam alguns desafios a serem enfrentados pelos profissionais para prestar um atendimento integral ao neonato. Dentre esses, maior valorização e incentivo à participação da família no processo assistencial, efetivação do trabalho em equipe, especialmente articulado em torno de um projeto assistencial comum e mudanças nas inter-relações entre os profissionais e usuários da unidade.

Considerando a saúde da criança como área estratégica de atuação da equipe de saúde da família, a realização de outros estudos que avaliem a assistência ao recém-nascido/neonato se torna importante, visto que este analisou apenas uma equipe da ESF do município de estudo. Além disso, o conhecimento sobre como vem se efetivando a atenção a esse grupo populacional em nossa realidade pode oferecer subsídios para a melhoria da qualidade da assistência prestada ao neonato nas unidades básicas de saúde, garantindo o direito que estas crianças têm a um atendimento integral, resolutivo e de qualidade.

\section{REFERÊNCIAS}

1. Santos Neto ET, Alves KCG, Zorzal M, Lima, R de CD. Políticas de Saúde Materna no Brasil: os nexos com indicadores de saúde materno-infantil. Saúde soc. [Internet] 2008;17(2) [acesso em 29 nov 2012]. Disponível: http://www.scielo.br/scielo.php?script=sci_ issuetoc\& pid=0104-129020080002\&lng=pt\&nrm $=$ iso

2. Costa R, Padilha MI, Monticelli M, Ramos FRS, Borenstein. Políticas públicas de saúde ao recémnascido no Brasil: reflexos para a assistência neonatal, história da enfermagem. Hist. Enf. Rev. Eletr. (HERE). [Internet] 2010;1(1) [acesso em 09 nov 2012] Disponível: http://ww.abennacional.org.br/centrodememoria/here/ n1vollano1_artigo4.pdf

3. Ministério da Saúde (BR). Secretaria de Atenção à Saúde. Departamento de Ações Programáticas Estratégicas. Agenda de compromissos para a saúde integral da criança e redução de mortalidade infantil. Série e normas e manuais técnicos. Brasília (DF), 2004.

4. Furtado MCC, Mello DF, Parada CMGL, Pinto IC, Reis MCG, Scochi CGS. Avaliação da atenção ao recém-nascido na articulação entre maternidade e rede básica de saúde Rev. Eletr. Enf. [Internet] 2010;12(4) [acesso em 05 nov 2012] Disponível: http://www.fen. ufg.br/revista/v12/n4/v12n4a07.htm. doi: 10.5216/ree. v12i4.7625

5. Constituição da República Federativa do Brasil. Brasília (Brasil): Senado Federal; 1988.

6. Del Ciampo LA, Ricco RG, Daneluzzi JC, Del Ciampo IRL, Ferraz IS, Almeida CAN. O programa de saúde da família e a puericultura. Ciênc. saúde colet. [Internet] 2006;11(3) [acesso em 29 nov 2012]. Disponível: http://www.scielo. br/scielo.php?script $=$ sci_issuetoc $\&$ pid $=1413$ $812320060003 \& \operatorname{lng}=$ pt\&nrm $=$ iso

7. Dias NS. Assistência ao neonato em uma unidade de saúde da família no município de Cuiabá-MT [dissertação]. Cuiabá: Universidade Federal de Mato Grosso; 2008.

8. Dias NS, Gaiva MAM. Assistência ao neonato em uma unidade de saúde da família no município de CuiabáMT. Nursing. 2010;13(148):474-9.

9. Gomes, R. Análise e interpretação de dados em pesquisa qualitativa. In: Minayo MCS, organizadora. Pesquisa social: teoria método e criatividade. $25^{\mathrm{a}}$ ed. Petrópolis (RJ): Vozes; 2007. p. 79-108. 
10. Ministério da Saúde (BR). Secretaria de atenção à saúde. Política Nacional de Atenção Básica. Portaria ${ }^{\circ}$ 648, de 28 de março de 2006 [Internet] Brasília (DF): Ministério da Saúde; 2006 [acesso em 10 nov 2012]. Disponível: http://dtr2004.saude.gov.br/dab/legislacao/ portaria_648_28_03_2006.pdf. 2009.

11. Campos, GWS. Considerações sobre a arte e a ciência da mudança: revolução das coisas e reforma das pessoas. $\mathrm{O}$ caso da saúde. In: Cecilio LCO, organizador. Inventando a mudança na saúde. $2^{a}$ ed. São Paulo: Hucitec; 1997. p. 29-87.

12. Ministério da Saúde (BR). Secretaria de Políticas Públicas. Guia prático do Programa de Saúde da Família. Brasília (DF); 2001.

13. Ministério da Saúde (BR). Secretaria de Políticas de Saúde. Saúde da criança: acompanhamento do crescimento e desenvolvimento infantil. Brasília (DF); 2002.

14. Lima IMSO, Alves VS, Franco ALS. A consulta médica no contexto do PSF e o direito da criança. Rev. bras. crescimento desenvolv. hum. [Internet] 2007;17(3) [acesso em 29 nov 2012]. Disponível: http:// www.revistasusp.sibi.usp.br/scielo.php?script $=$ sci issuetoc\&pid=0104-128220070003\&lng=pt\&nrm=iso

15. Carvalho MF, Lira PIC, Romani SAM, Santos IS, Veras AACA, Batista Filho M. Acompanhamento do crescimento em crianças menores de um ano: situação nos serviços de saúde em Pernambuco, Brasil. Cad. Saúde Pública. [Internet] 2008;24(3) [acesso em 29 nov 2012]. Disponível: http://www. scielo.br/scielo.php?script $=$ sci_issuetoc $\&$ pid $=0102-$ 311X20080003\&lng=pt\&nrm=iso

16. Mandú ENT, Gaíva MAM, Silva M da A, Silva AMN da. A visita domiciliária sob o olhar de usuários do programa saúde da família. Texto Contexto Enferm. [Internet] 2008;17(1) [acesso em 29 nov 2012]. Disponível: http://www.scielo.br/scielo.php?script=sci issuetoc\&pid $=0104-070720080001 \& \operatorname{lng}=$ pt\&nrm=iso

17. Oliveira AR, Chaves AEP, Nogueira JA, Sá LD, Collet N. Satisfação e limitação no cotidiano de trabalho do agente comunitário de saúde. Rev. Eletr. Enf. [Internet] 2010;12(1) [acesso em 09 nov 2012]. Disponível: http:// www.fen.ufg.br/revista/v12/n1/v12nla04.htm

18. Oliveira GP, Peres AM, Chaves MMP, Eduardo EA. Os desafios para a intervenção em saúde na estratégia de saúde da família segundo os profissionais. Cogitare enferm. [Internet] 2011;16(3) [acesso em 29 nov 2012].
Disponível: http://ojs.c3sl.ufpr.br/ojs2/index.php/ cogitare/issue/view/1252

19. Araújo MBS, Rocha PM. Trabalho em equipe: um desafio para a consolidação da estratégia de saúde da família. Ciênc. saúde colet. [Internet] 2007;12(2) [acesso em 09 nov 2012]. Disponível: http://www.scielo.br/pdf/ $\mathrm{csc} / \mathrm{v} 12 \mathrm{n} 2 / \mathrm{a} 22 \mathrm{v} 12 \mathrm{n} 2 . \mathrm{pdf}$

20. Ramos, D. D; Lima, M. A. D. S. Acesso e acolhimento aos usuários em uma unidade de saúde de Porto Alegre, RS, Brasil. Cad. Saúde Pública. [Internet] 2003;19(1) [acesso em 29 nov 2012]. Disponível: http://www.scielo. br/pdf/csp/v19n1/14902.pdf 